\title{
Neurofunctional changes after a single mirror therapy intervention in chronic ischemic stroke
}

\author{
Morgana Novaes, Fernanda Palhano-Fontes, Andre Peres, Kelley Mazzetto- \\ Betti, Maristela Pelicioni, Kátia Andrade, Antonio Santos, Octavio Pontes- \\ Neto \& Draulio Araujo
}

To cite this article: Morgana Novaes, Fernanda Palhano-Fontes, Andre Peres, Kelley MazzettoBetti, Maristela Pelicioni, Kátia Andrade, Antonio Santos, Octavio Pontes-Neto \& Draulio Araujo (2018): Neurofunctional changes after a single mirror therapy intervention in chronic ischemic stroke, International Journal of Neuroscience, DOI: 10.1080/00207454.2018.1447571

To link to this article: https://doi.org/10.1080/00207454.2018.1447571

Accepted author version posted online: 01

Mar 2018.

Submit your article to this journal $[\pi$

Q View related articles $₫$

View Crossmark data \lceil 
Publisher: Taylor \& Francis

Journal: International Journal of Neuroscience

DOI: https://doi.org/10.1080/00207454.2018.1447571

\section{Neurofunctional changes after a single mirror therapy intervention in} chronic ischemic stroke

Novaes, Morgana ${ }^{1^{*}}$; Palhano-Fontes, Fernanda ${ }^{1^{*}}$; Peres, Andire ${ }^{\frac{1}{3}}$ Mazzetto-

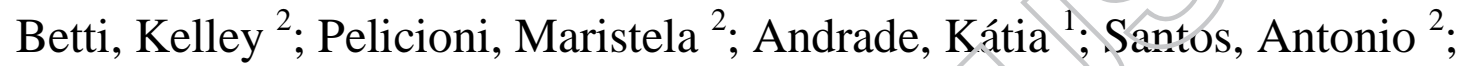

Pontes-Neto, Octavio ${ }^{2}$; Araujo, Draulio'.

${ }^{1}$ Brain Institute/Onofre Lopes University Hospital, Federal Jniversity of Rio Grande do Norte (UFRN), Natal-RN, Brazil. 'Department of Neuroscience and Behavioral Sciences, Ribeirão Preto School of Medicine, University of Sao Paulo (USP), Ribeidı ล̃o Preto-SP, Brazil.

*These authors contributed equally to the study

Corresponding author:

Prof. Dráulio B. de Araújo

Brain Institute (UFRN).

Av. Nascimento de Castro, 2155.

59056-450 - Nata1/RN, Brazil.

Phone. +55 84 3215-2709

e-mail:draulis@neuro.ufrn.br

Abstract

Background: Mirror therapy (MT) is becoming an alternative rehabilitation strategy for various

conditions, including stroke. Although recent studies suggest the positive benefit of MT in chronic stroke motor recovery, little is known about its neural mechanisms. Purpose: To identify functional brain changes induced by 
a single MT intervention in ischemic stroke survivors, assessed by both Transcranial Magnetic Stimulation (TMS) and functional Magnetic Resonance Imaging (fMRI). Materials and Methods: TMS and fMRI were used to investigate fifteen stroke survivors immediately before and after a single thirty minutes MT session. Results: We found statistically significant increase in post-MT MEP amplitude (increased excitability) from the affected primary motor cortex (M1), when compared to pre-MT MEP. Post-MT fMRI maps were associated with a more organized and constrained pattern, with a more focal M1 activity within the affected hemisphere after MT, limited to the cortical area of hand representation. Furthermore, we find a change in the balance of M1 activity toward the affected hemisphere. In addition, significant correlation was found between derreased fiMRI 5 values and increased Motor Evoked Potential (MEP) amplitude post-MT, in the affected hemisphere. Conclusion: Our study suggests that a single MT intervention in stroke survivors is reiated to increased MEP of the affected limb, and a more constrained activity of the affected M1, as if activity had become more constrained and limited to the affected hemisphere.

Keywords: Mirror therapy, ischemic streke, Stroke rehabilitation, Transcranial magnetic stimulation, functional Magnetic Resonance Imaging.

\section{Introduction}

Ischemic stroke is frequently accompanied by motor functions deficits, which evolves with limited spontaneous recevery particularly at the chronic stage of stroke (6 months after the insult). ${ }^{1,2}$ During this stage, patients usually undergo motor rehabilitation programs ${ }^{3-6}$

A promising rehabilitation approach is the use of mirror therapy (MT), which was originally designed and used to help alleviating phantom limb pain. ${ }^{7}$ More recently, it has been tested as an adjuvant therapy in stroke motor rehabilitation..$^{8-11}$ During an MT session for stroke, patients place their hands in a mirror box, composed of two compartments separated by a vertical mirror (sup. fig. 1). The affected hand is positioned in one side of the box, behind the mirror, and the unaffected hand in the other side, so the reflection of the unaffected hand is seen in the place of the affected one (supp. 
fig. 1). Patients are oriented to looks at the mirror and are instructed to move their unaffected hand, creating the illusion that the affected hand is moving.

Most studies that tested MT in stroke rehabilitation have observed improved motor performance after 6-8 weeks of MT-based programs. ${ }^{9,10,12}$ For instance, a recent randomized controlled trial suggests that 8 weeks of MT (40 sessions, 5/week) promotes significant improvement in motor function, when compared to standard motor rehabilitation. ${ }^{9}$ Nevertheless, the underlying neural mechanisms related to the motor recovery induced by MT are still unclear.

Functional Magnetic Resonance Imaging (fMRI) studies suggest that brain reorganization is not limited to the motor system, but also involves changes in the parieto-occipita1 lobe, and dorsal frontal areas. ${ }^{13}$ The most consistent finding, however, is that impreved motor performance after MTbased programs are related to increased activity of the affected primary motor cortex (M1), as if MT changes the balance of M1 activity between hemispheres, toward the affected hemisphere..$^{10,14,15}$

There are also a few Transcranial Magnetic Strmulation (TMS) studies designed to understand the neural basis of MT interventions, and most of them have been conducted in healthy subjects. A consistent observation is an increased excitability of ipsilateral M1 (to the moving hand), both during the MT session, ${ }^{16-18}$ and immediateiy after the session. ${ }^{19}$ There is only one study in stroke survivors which used TMS stimuli daring the MT execution. ${ }^{20}$ The results suggest increased excitability of the affected M1, with respect to the unaffected M1. ${ }^{20}$

St 1 dies conducted in stroke so far have assessed MT-induced changes by TMS or fMRI, separaty, and after the completion of many MT sessions (fMRI studies) or during MT execution (TMS studies). However, it is still not clear if MT neural changes already occur at the first hours after a sing $\mathrm{MT}$ training. Therefore, this study sought to investigate MT sub-acute changes in the motor cortex of chronic stroke patients by both TMS and fMRI, and to explore the correspondences between them. Our initial hypothesis is that a single MT session would induce the following sub-acute changes: (i) increase TMS-MEP amplitude when the affected M1 is stimulated; (ii) increase fMRI activity in 
the affected M1; (iii) changes in fMRI M1 activity correlates with TMS-MEP changes observed in the affected hemisphere.

\section{Materials and Methods}

\section{Participant}

The Ethics and Research Committee of the Ribeirão Preto School of Medicine, University of São Paulo approved the study (\#860004294-11). All subjects provided written informed consent prior to their participation in the study.

Ischemic stroke survivors were selected from the Ribeirão Pre o Stroke R.egistry, according to the following inclusion criteria: (i) single ischemic stroke episode confirmed by computed tomography (CT) and/or magnetic resonance imaging (MRI), (ii) chronic stroke (more than 6 months after insult). (iii) be able to perform the fMRI task of opening and closing the hand. Participants were excluded if they had a general contraindication for TMS or fMIRI assessments, such as: cardiac pacemaker, aneurysm clips, carotid or cerebral stents, metal implants, pregnancy.

Demographic and clinical data are presented in tables 1 and 2. Most participants were righthanded $(93 \%, \mathrm{~N}=14)$, male $(57 \%, \mathrm{~N}=10)$, adults $(58 \pm 10$ y.o). All were stable in terms of neurological deficits. On average, the ischemic insult had occurred 25 months before the study, and in most cases (53\%) compromised the left middle cerebral artery (MCA)

\section{Clinical instruanents}

Clinical evaluation was used to characterize the upper limb motor function. The following tests were uscé, only at baseline (before MT): (i) Box and block test to assess gross manual dexterity. ${ }^{21}$ This test evaluates the number of blocks moved in 60 seconds from one compartment of the box to the other; (ii) Purdue Pegboard test to assess fine manual dexterity. ${ }^{22}$ This test evaluates the number of pins placed into small holes in 30 seconds; (iii) Action Research Arm Test (ARAT) to evaluate 
compression activities, handgrip, clamping and outreach activities, ${ }^{23}$ (iv) Fugl-Meyer Assessment (FMA) for the upper limbs to assess sensitivity, motor function, speed and coordination of the upper $\operatorname{limbs}^{24}$

\section{Mirror Therapy intervention}

During the MT session, we instructed participants to position their affected hand in one side of the mirror box, behind the mirror, and the unaffected hand on the other side, in front of the inimor (supp fig. 1). Participants were instructed to look at the reflected image of the unaffected hard while performing the following series of movements with the unaffected hand (surpi. fig. 1) abductionadduction, flexion-extension, opponency of fingers with the thumb, an i prenation-supination of the forearm. The intervention lasted 30 minutes. Participants were monitored to ascertain correct task execution, and to evaluate synkinesis of the affected hand, which was not supposed to move.

\section{Experimental protocol}

This manuscript is a descriptive study in which fiftcen patients were included and evaluated before and after Mirror Therapy by scales. TMIS and fMRI. Figure 1 shows a diagram of the experimental protocol. Assessments occurred on three separate days. At the first encounter, patients were submitted to a clinical neurological evaluation, and were submitted to the box of block test, the Purdue test, the ARAT, and the FMA assessments. At this same day, participants were randomized with respect to the order of the assessments: fMRI or TMS first.

TMS and MRI sessions occurred on two separate days. In each day, TMS or fMRI was conducted inmediately before and immediately after a 30 minutes MT intervention. In each of these sessions, patients enrolled in a baseline assessment (TMS or fMRI), followed by the MT intervention, and by a post-MT assessment (TMS or fMRI) (Fig. 1). Both evaluations (TMS and fMRI) were performed within a week. 


\section{TMS session}

TMS stimulation was delivered to the affected hemisphere only, and Motor Evoked Potentials (MEP) were acquired on the affected hand only. We used a NeuroSoft TMS stimulator (Neuro-MS, Russia), with a butterfly coil (larger/smaller radius $=20 \mathrm{~cm} / 10 \mathrm{~cm}$ ) positioned over the primary motor cortex (M1) at an angle of $\sim 45^{\circ}$ with respect to the longitudinal fissure. MEPs were detected with two siiversurface electrodes, one positioned over the abductor muscle of the thumb, referenced against the one positioned over the prominence of the seventh cervical vertebra. Signals were amplified and conditioned by a Myosystem (12-bit A/D Converter) electromyography system, with a sampling rate of $4000 \mathrm{~Hz}$, and a band pass filter set at 20-1000 Hz.

Individual hot spots were determined as the site of stimulation that evokes the largest MEP amplitude. Individual motor threshold was determined as the minima! stimulation intensity that evokes MEP responses of at least $50 \mu \mathrm{V}$ in five out of 10 consecutive TMis pulses. ${ }^{25}$ MEP response was composed by the average of ten consecutive TMS puises applied to the individual hot spot at $110 \%$ of the individual motor threshold, randomly distribated across a 60 seconds interval. The TMS protocol was applied when the subjects were at rest.

\section{fMRI session}

Magnetic resonance images (MRI) were collected in a 3T scanner (Philips, Achieva, The Netherlands). fNiRI statistical maps were based on an Echo Planar Image (EPI) sequence with the following accrisition parameters: TR/TE $=2000 / 60 \mathrm{~ms}$, flip angle $=90^{\circ}, \mathrm{FOV}=230 \mathrm{~mm}$, slice thickness= $3 \mathrm{~mm}$, 30 slices, 118 volumes. A set of high-resolution 3D T1-weighted anatomical images $\left(1 \mathrm{~mm}^{3}\right)$ were also obtained, with the following parameters: $\mathrm{TR}=5.7 \mathrm{~ms}$; TE $=2.6 \mathrm{~ms}$; flip angle $=8^{\circ}$, matrix $=256 \times 256, \mathrm{FOV}=256 \mathrm{~mm}$, slice thickness $=1 \mathrm{~mm}$.

The fMRI motor task consisted of opening and closing the affected hand at self-pace. The chosen protocol used an event-related design to avoid habituation effects, commonly observed in stroke. ${ }^{26}$ The protocol encompassed alternating periods of movement (6s) with periods of rest (16s). 
Auditory cues were delivered to signal a change between conditions. In order to assure task compliance, participants performed a dry run before fMRI acquisition.

\section{Data Analysis}

\section{Scales}

Statistical analysis of the scales was performed with a two-tailed Wilcoxon test (data is not normally distributed), using the GraphPad Prism software (GraphPad Software v5.0, San Diego, USA.), comparing scores from the affected against the unaffected hand at baseline. We expressed vaiues as median, Z-value and statistical significance was set at $\mathrm{p}<0.05$.

\section{TMS}

MEP analysis was conducted in MATLAB 7.10. Group analysis compared the grand median MEP values (pre-MT vs post-MT), obtained from the 10 indi idual MLPs detected before and after the MT intervention. A Wilcoxon test (data is not normally distributed) was used to compare MEPs from the two endpoints (before $\mathrm{x}$ after MT). Statistical threshoid was set at $\mathrm{p}<0.05$ (two-tailed). We expressed values as median, $\mathrm{Z}$ value and stat stical significance was set at $\mathrm{p}<0.05$.

For individual subject inspection, the 10 detected MEPs for each subject were compared (preMT vs post-MT) using the Miann-Whitney test, since the data is not normally distributed and the samples are indeperidert. We expressed values as median, U-value and statistical significance was set at $\mathrm{p}<0.05$.

\section{fMRI}

flMR preprocessing and statistical analyses were performed in SPM12 (Statistical Parametric Mapping, Wellcome Trust Centre for Neuroimaging, UK). Preprocessing included slice-time correction, head motion correction, spatial smoothing (Gaussian kernel, FWHM=5 mm), and a highpass filter set at $0.02 \mathrm{~Hz}$. Images were normalized to the MNI152 template. To have all lesions falling 
onto the same side, participants with right-sided lesions had their images flipped about the mid-sagittal plane.

First-level individual analysis used the general linear model (GLM) with a single regressor of interest modeling task periods. A single regressor was obtained by convolving a boxcar function of the task periods with a double-gamma hemodynamic response function. The six motion parameters (three rotations and three translations) were included in the model as regressors of no interest. Contrast images were obtained for each subject for the task $>$ rest condition using a $p<0.05$, uncorrecied. These contrast images were used in a subsequent second-level group analysis to examirie differences between pre- and post-MT. Whole brain analysis was conducted with a smalivolume correction at two regions of interest (ROI): the bilateral primary motor cortex (M1) and Suppleinentary Motor Area (SMA). These two ROI were extracted from the sensorimotor netvork atlas. ${ }^{27}$ Contrast maps were calculated with respect to: (i) pre-MT; (ii) post-MT; (iii) pre-MT > post-MT and; (iv) post-MT > preMT. Statistical threshold was set at $\mathrm{p}<0.05$, and clusters with. $\mathrm{p}_{\text {f }}<0.01$ at peak level.

\section{fMRI x TMS correlation}

To evaluate the correspondence between TMIS and fMRI changes elicited by MT, Pearson's correlation coefficient was calculated between individual M1 fMRI $\beta$-values changes ( $\left.\frac{\text { post_MT - pre_MT }}{\text { pre_MT }}\right)$, and the mean MEP amplitude changes $\left(\frac{\text { post_MT - pre_MT }}{\text { pre_MT }}\right)$ from the affected hemisphere. Individual fMRI $\beta$ values were extracted from a mask created by the intersection between the individual first-level statistical mans, before the MT intervention, and the M1 ROI defined in the sensorimotor network atlas.

\section{Results}

Fifteen patients were included in the study. Six participants performed the fMRI assessments first, and in nine TMS was applied first (table 2). Two participants had to be excluded from fMRI analysis: one due to significant hearing impairment, and the other due to claustrophobia. Five subjects had to be 
excluded from TMS analysis: in two, no MEP was detected, in the other two, EMG signal was too noisy, and one patient did not complete the post-MT session. fMRI was successfully conducted in 13 subjects, TMS in 10 subjects, and 8 patients were successfully submitted to both fMRI and TMS analysis.

Individual results for the ARAT, Fugl-Meyer, box of blocks and Purdue assessments are presented in table 2. Motor deficits observed at initial clinical evaluation involved mainly subtle aspects of movements, such as fine manual dexterity (Purdue test, $\mathrm{p}=0.02, \mathrm{Z}=-2.29, \Delta$ median $=3$ ), sensitivity and coordination (Fugl-Meyer, $\mathrm{p}=0.002, \mathrm{Z}=-3.06, \Delta$ median=2) $($ Table 1$)$. We did not observe statistically significant differences in the ARAT, nor in the box of biocks tests from the affected hand, when compared to unaffected hand (Table 1).

Figure 2a shows the median MEP detected in the affected hand before and after MT. We found significantly increased MEP amplitude (increased excitability) from the affected M1 post-MT, when compared to pre-MT (Fig. 2a, p=0.03, $\mathrm{Z}=-2,19$, median difference=0.25). Median values changed from $0.88 \mathrm{mV}$ to $1.41 \mathrm{mV}$. Figure $2 \mathrm{~b}$ shows the MEP changes ( $\triangle \mathrm{MEP}$ ) observed at the individual subject level. MEP ampliture measured in the affected hand of four participants (marked in -black) increased significantly after therapy (Fig. $2 b, p<0.05$ ). Analytical individual MEP results from all participants are preserted in table 3 .

Figure 3 shows group level fMRI results, pre- and post-MT. The motor task engaged the bilateral Mi and SMA, both before and after MT (Fig. 3). Figure 4 shows the between session comparison (post-MT vs pre-MT). MT led to changes of the primary motor cortex (M1). Post-MT IIVRI maps yere associated with a more organized and constrained pattern, with a more focal M1 activity within the affected hemisphere after MT, limited to the area of hand representation (Fig. 4). Furthermore, we find a change in the balance of M1 activity toward the affected hemisphere. At the individual level, the same patterns of reorganization were observed in 10 participants (77\%) (suppl. fig. 2). 
Figure 5 shows a significant Pearson's correlation between individual fMRI $\beta$-values changes with respect to individual amplitude MEP change in the affected M1.

\section{Discussion}

In this study we used both TMS and fMRI to investigate sub-acute neural changes induced by a single MT intervention in ischemic stroke survivors. At the group level, TMS revealed increased exeltability of the affected M1, observed as increased MEP amplitude post-MT, with respect to pre-MT. AMRI reveals a change in the balance of M1 activity toward the affected hemisphere, with increased fMRI focal activity post-MT in the affected M1, and a decreased activity in the unatfected M1 We also found a significant correlation between increased MEP amplitude and decreased iRI $\beta$-values in the affected M1.

Changes in MEP status are frequently observed in stroke, with decreased MEP amplitude (decreased excitability) of the affected M1 with respect to the unaffected M1 ${ }^{28-31}$ In fact, the TMS excitability of the affected M1 is an importat marker of motor recovery after stroke. ${ }^{30,32,33}$ For instance, evidence suggest that the MEP amplitude from the affected M1 measured one day after an ischemic stroke is predictive of the motor recovery observed 14 days after the insult. ${ }^{30}$ Presumably, the excess of transcallosal inhibitiory activity from the unaffected M1 to the affected hemisphere compromises the moter recovery of these patients. ${ }^{34-37}$

In line with these findings, an increasing number of studies have suggested that increased fMRI activity of the unaffected M1, with respect to the affected M1, is associated with poorer motor reco ery. ${ }^{6,10}, 38-42$ On the contrary, we found significant increased focal fMRI activity in the affected M1, and decreased activity in the unaffected M1, after a single MT intervention, with a more restricted M1 activity. ${ }^{43-45}$ Furthermore, increasing evidence points to a widespread recruitment of brain regions early after the stroke insult, followed by a progressive reduction in this task-related recruitment over the course of rehabilitation sessions. ${ }^{6,39,46}$ For instance, an fMRI study in stroke observed a linear decrease in the size of unaffected M1 activity, along the course of a 2 weeks rehabilitation program 
with constraint-induced movement therapy, which correlated with the observed motor improvement. ${ }^{39}$ Also, rehabilitation programs with functional and/or nonfunctional exercises observed decreased sparseness in fMRI activity maps, reflecting a decreased activity of the unaffected M1, decreased perilesional M1 activity. ${ }^{6}$

These sub-acute MT modifications are consistent with previous fMRI studies that found changes in the motor cortex after many MT session. ${ }^{10,14}$ For example, previous studies in stroke survivors suggests that the improved motor performance after 30-40 MT sessions is accompanied by increased activity of the affected M1..$^{10,14}$ Overall, these results suggest that a single MT intervention may already reduce excessive transcallosal inhibitory activity from the unaffected hiemisphere over the affected one. ${ }^{37}$

Individual subject variability is generally high for both TMS and IMRI evaluations and, therefore, most studies restrict their analysis to the gro $\mathrm{p}$ level. To draw reader's attention to the current clinical relevance of our study, the results are also presented at the individual subject level. Significant increased MEP amplitudes were ebs rved pcst-MT in $40 \%$ of the participants. MEP did not decrease for any subject. Congruent rocal increased activity of the affected M1, and decreased activity of the unaffected M1 was identified in 54\% of the participants. It is possible that these changes become more consistent with increasing number of interventions. Furthermore, such variability may be an important early narker of the clinical benefit specific patients might expect after an MT-based rehabilitation piograin.

We also foynd a significant correlation between increased MEP amplitudes and decreased $\beta$ values extracted from the affected M1 ROI. Increased MEP amplitude was significantly correlated with decreased $\beta$-values after MT. Although the correspondences between fMRI and TMS variables aire difficult to trace, it has been observed that patients with more pronounced fMRI activity in the affected hemisphere also had a more balanced transcallosal inhibition. ${ }^{37}$ 
Our results bring preliminary evidence of the beneficial effect of a single MT session.

However, it is important to point out some limitations and caveats of our study: (i) the absence of a control group (without intervention), which limits our ability to associate the observed changes to the MT intervention; (ii) the number of patients is small; (iii) we did not evaluate patient's clinical improvement; (iv) the cohort was restricted to well-recovered patients, with mild motor impairment, as study participants should be able to move their hand during the fMRI protocol; (v) the use of fixedeffects models, which limits the conclusion of our results to the population studied; (vi) it is know that within-session fMRI reliability is higher than between-session, particularly in stroke starvivors who show altered cerebrovascular coupling, which may interfere with fMRI analysis and interpretation 26,47,48;

Overall, our study suggests that a single MT intervention in stroke survivors is related to increased MEP of the affected limb, a more constrained activity of the affected M1, a correspondence between the observed changes due to MT upon FMRI and TMS parameters, as if activity had become more constrained and limited to the affected hemisphere. This work contributes to better understand the neural mechanisms of MT in stroke survivors. Besides, exposing individual data may allow other researchers to find similarities with our data and contribute to understanding nuances of MT intervention in stroke survivors.

\section{Acknowledgements}

The authors vould like to express their gratitude to all patients who volunteered for this experiment, and to Proi Antonio Pereira e Tania Campos for the fruitful discussions.

\section{Disclosure of interest}

The authors report no conflicts of interest.

\section{Funding details}


This work was supported by the Brazilian National Council for Scientific and Technological Development (CNPq) [grants number 479466/2013 and 466760/2014], and by the CAPES Foundation within the Ministry of Education [grants number 1677/2012 \& 1577/2013]. The authors declare no competing financial interests.

\section{References}

1. Cramer SC. Repairing the human brain after stroke: I. Mechanisms of spontaneous recovery. Ann Neurol. 2008;63(3):272-287. doi:10.1002/ana.21393.

2. Johansson BB. Current trends in stroke rehabilitation. A review with focus on brain plasticity. Acta Neurol Scand. 2011;123(3):147-159.

3. Allman C, Amadi U, Winkler AM, Wilkins L, Filippini N, Rischkà U, Stagg CJ, JohansenBerg H. Ipsilesional anodal tDCS enhances the functionai benefits of rehabilitation in patients after stroke. Sci Transl Med. 2016;8(330):330re1-330re idei-10.1126/scitranslmed.aad5651.

4. Park JH. The effects of modified constraint induced therapy combined with mental practice on patients with chronic stroke. J Phys Ther Sci.2015:27(5):1585.

5. Park J. Influence of mental practice on upper limb muscle activity and activities of daily living in chronic stroke patients. J Phys Ther Sci. 2016;28(3):1061.

6. Pelicioni MCX, Novaes Man, Peres ASC, Lino De Souza AA, Minelli C, Fabio SRC, PontesNeto OM, Santos AC, De Araujo DB. Functional versus nonfunctional rehabilitation in chronic ischemic stroke. Evidences from a randomized functional MRI study. Neural Plast. 2016;2016. doi: $10.1155 ! 201 \% / 6353218$.

7. Ramachandran VS, Rogers-Ramachandran D. Synaesthesia in phantom limbs induced with Mirrors. Proc Biol Sci. 1996;263(1369):377-386. doi:10.1098/rspb.1996.0058.

Altschuler EL, Wisdom SB, Stone L, Foster C, Galasko D, Llewellyn DME, Ramachandran VS. Rehabilitation of hemiparesis after stroke with a mirror. Lancet. 1999;353(9169):20352036.

9. Arya KN, Pandian S, Kumar D, Puri V. Task-Based Mirror Therapy Augmenting Motor Recovery in Poststroke Hemiparesis: A Randomized Controlled Trial. J Stroke Cerebrovasc Dis. 2015;24(8):1738-1748. doi:10.1016/j.jstrokecerebrovasdis.2015.03.026. 
10. Michielsen ME, Selles RW, van der Geest JN, Eckhardt M, Yavuzer G, Stam HJ, Smits M, Ribbers GM, Bussmann JBJ. Motor Recovery and Cortical Reorganization After Mirror Therapy in Chronic Stroke Patients: A Phase II Randomized Controlled Trial. Neurorehabil Neural Repair. 2011;25(3):223-233. doi:10.1177/1545968310385127|10.1177/1545968310385127.

11. Sathian K, Greenspan AI, Wolf SL. Doing it with mirrors: A case study of a novel approach to neurorehabilitation. Neurorehabil Neural Repair. 2000;14(1):73-76. doi:10.1177/154596830001400109.

12. Thieme H, Mehrholz J, Pohl M, Behrens J, Dohle C. Mirror therapy for improving motor function after stroke. Stroke. 2013;44(1). doi:10.1161/STROKEAHA.112.673087.

13. Arya K. Underlying neural mechanisms of mirror therapy: Implications for motor rehabilitation in stroke. Neurol India. 2016;64(1):38. doi:10.4103/0028-3886.173622.

14. Bhasin A, Bhatia R, Kumaran S, Mohanty S, Padma Srivastava M. Neural interface of mirror therapy in chronic stroke patients: A functional magnetic resonance imaging study. Neurol India. 2012;60(6):570. doi:10.4103/0028-3886.105188.

15. Michielsen ME, Smits M, Ribbers GM Stam HJ vah der Geest JN, Bussmann JBJ, Selles RW. The neuronal correlates of mirror therapy: an fiMRI study on mirror induced visual illusions in patients with stroke. J Neurol Neurosurg Psychiatry. 2011;82(4):393-398. doi:10.1136/jnnp.2009.194134.

16. Fukumura K, Sugayara K. Tailabe S, Ushiba J, Tomita Y. Influence of mirror therapy on human motor cortex. Int $\boldsymbol{j}$ Neurosci. 2007;117(7):1039-1048. doi:10.1085/0020745e600936841|10.1080/00207450600936841.

17. Funase K, Tàbıra T, Higashi T, Liang N, Kasai T. Increased corticospinal excitability during direct observation of self-movement and indirect observation with a mirror box. Neurosci Lett. 2007;419(2):108-112. doi:S0304-3940(07)00453-3 [pii] 10.1016/j.neulet.2007.04.025.

18. Garry MI, Loftus A, Summers JJ. Mirror, mirror on the wall: viewing a mirror reflection of unilateral hand movements facilitates ipsilateral M1 excitability. Exp Brain Res. 2005;163(1):118-122. doi:10.1007/s00221-005-2226-9|10.1007/s00221-005-2226-9.

19. Nojima I, Mima T, Koganemaru S, Thabit MN, Fukuyama H, Kawamata T. Human Motor Plasticity Induced by Mirror Visual Feedback. J Neurosci. 2012;32(4):1293-1300. doi:10.1523/JNEUROSCI.5364-11.2012. 
20. Kang YJ, Ku J, Kim HJ, Park HK. Facilitation of corticospinal excitability according to motor imagery and mirror therapy in healthy subjects and stroke patients. Ann Rehabil Med. 2011;35(6):747-758. doi:10.5535/arm.2011.35.6.747.

21. Desrosiers J, Bravo G, Hébert R, Dutil E, Mercier L. Validation of the Box and Block Test as a measure of dexterity of elderly people: reliability, validity, and norms studies. Arch Phys Med Rehabil. 1994;75(7):751-755. doi:0003-9993(94)90130-9 [pii].

22. Desrosiers J, Bourbonnais D, Bravo G, Roy PM, Guay M. Performance of the "unaffected" upper extremity of elderly stroke patients. Stroke. 1996;27(9):1564-1570.

23. Van der Lee JH, De Groot V, Beckerman H, Wagenaar RC, Lankhorst GJ, Bouter L M, der Lee JH, De Groot V, Beckerman H, Wagenaar RC, Lankhorst GJ, Bouter LM. The irtya- and interrater reliability of the action research arm test: A practical test of upner extremity function in patients with stroke. Arch Phys Med Rehabil. 2001;82(1):14-19. doi:10.1053/apmr.2001.18668.

24. Fugl-Meyer AR, Jääskö L, Leyman I, Olsson S, Stegliné S. The post-stroke hemiplegic patient. 1. a method for evaluation of physical performance Scand J Rehabil Med. 1975;7(1):13-31. doi:10.1038/35081184.

25. Conforto AB, Z'Graggen WJ, Kohl AS, Resler KM, Kaelin-Lang A. Impact of coil position and electrophysiological monitoring on determination of motor thresholds to transcranial magnetic stimulation. Clin Neirophy iol. 2004;115(4):812-819. doi:10.1016/j.clinph.2503.11.010]10.1016/j.clinph.2003.11.010.

26. Mazzetto-Betti KC, Leoni RF, Pontes-Neto OM, Santos AC, Leite JP, Silva AC, De Araujo DB. The stability of the blood oxygenation level-dependent functional MRI response to motor tasks is oltered in patients with chronic ischemic stroke. Stroke. 2010;41(9):1921-1926. doi:10.1151/STROKEAHA.110.590471.

27. Shirer WR, Ryali S, Rykhlevskaia E, Menon V, Greicius MD. Decoding subject-driven cognitive states with whole-brain connectivity patterns. Cereb Cortex. 2012;22(1):158-165. doi:10.1093/cercor/bhr099.

28. Byrnes ML, Thickbroom GW, Phillips BA, Mastaglia FL. Long-term changes in motor cortical organisation after recovery from subcortical stroke. Brain Res. 2001;889(1-2):278-287. doi:S0006-8993(00)03089-4 [pii].

29. Escudero J V, Sancho J, Bautista D, Escudero M, López-Trigo J. Prognostic value of motor 
evoked potential obtained by transcranial magnetic brain stimulation in motor function recovery in patients with acute ischemic stroke. Stroke. 1998;29(9):1854-1859.

http://www.ncbi.nlm.nih.gov/pubmed/9731608.

30. Rapisarda G, Bastings E, de Noordhout AM, Pennisi G, Delwaide PJ. Can motor recovery in stroke patients be predicted by early transcranial magnetic stimulation? Stroke. 1996;27(12):2191-2196. http://www.ncbi.nlm.nih.gov/pubmed/8969779.

31. Liepert J, Storch P, Fritsch A, Weiller C. Motor cortex disinhibition in acute stroke. C!in Neurophysiol. 2000;111(4):671-676. doi:S1388-2457(99)00312-0 [pii].

32. Koski L, Mernar TJ, Dobkin BH. Immediate and long-term changes in corticomotor o:ıtput in response to rehabilitation: correlation with functional improvements in chronic stroke. Neurorehabil Neural Repair. 2004;18(4):230-249. doi:18/4/230 [pii] $10.1177 / 1545968304269210$.

33. Pennisi G, Rapisarda G, Bella R, Calabrese V, Maertens De Noordhout A, Delwaide PJ. Absence of response to early transcranial magnetic stimilation in ischemic stroke patients: prognostic value for hand motor recovery. Stroke. 1999-30:12):2666-2670. http://www.ncbi.nlm.nih.gov/pubmed/10582994.

34. Murase N, Duque J, Mazzocchio R, Ceheri IG. Influence of interhemispheric interactions on motor function in chronic stroke. Ann Neurol. 2004;55(3):400-409. doi:10.1002/ana.10848.

35. Shimizu T, Hosaki A, Hins T, Sats M, Komori T, Hirai S, Rossini PM. Motor cortical disinhibition in the unafiected nemisphere after unilateral cortical stroke. Brain. 2002;125(Pt 8):1896-1907. kttn://Www.nebi.nlm.nih.gov/pubmed/12135979.

36. Ward NS Cohen LG. Mechanisms underlying recovery of motor function after stroke. Arch Neurol.2004;61(12):1844-1848. doi:10.1001/archneur.61.12.1844.

37. Cunninghan D a, Machado A, Janini D, Varnerin N, Bonnett C, Yue G, Jones S, Lowe M, Beal $\mathrm{E}$, Sakaie K, Plow EB. Assessment of inter-hemispheric imbalance using imaging and noninvasive brain stimulation in patients with chronic stroke. Arch Phys Med Rehabil. 2015;96(4):S94-S103. doi:10.1016/j.apmr.2014.07.419.

38. Carey JR, Kimberley TJ, Lewis SM, Auerbach EJ, Dorsey L, Rundquist P, Ugurbil K. Analysis of fMRI and finger tracking training in subjects with chronic stroke. Brain. 2002;125(Pt 4):773-788. http://www.ncbi.nlm.nih.gov/pubmed/11912111.

39. Dong Y, Dobkin BH, Cen SY, Wu AD, Winstein CJ. Motor cortex activation during treatment 
may predict therapeutic gains in paretic hand function after stroke. Stroke. 2006;37(6):15521555. doi:01.STR.0000221281.69373.4e [pii] 10.1161/01.STR.0000221281.69373.4e.

40. Kim YH, Park JW, Ko MH, Jang SH, Lee PK. Plastic changes of motor network after constraint-induced movement therapy. Yonsei Med J. 2004;45(2):241-246. doi:200404241 [pii].

41. Levy CE, Nichols DS, Schmalbrock PM, Keller P, Chakeres DW. Functional MRI eviderice of cortical reorganization in upper-limb stroke hemiplegia treated with constraint-induced movement therapy. Am J Phys Med Rehabil. 2001;80(1):4-12. http://www.ncbi.nlm.nih.gov/pubmed/11138954.

42. Rossini PM, Dal Forno G. Neuronal post-stroke plasticity in the adult Restor Neurol Neurosci. 2004;22(3-5):193-206. http://www.ncbi.nlm.nih.gov/pubmed/15502265.

43. Cao Y, D'Olhaberriague L, Vikingstad EM, Levine SR, Welch KM. Pilot study of functional MRI to assess cerebral activation of motor function after postsiroke hemiparesis. Stroke. 1998;29(1):112-122. http://www.ncbi.nlm.nih.gov/pubrined/944.5338.

44. Cramer SC, Nelles G, Benson RR, Kaplan LD, Parker RA, Kwong KK, Kennedy DN, Finklestein SP, Rosen BR. A functional MRI study of subjects recovered from hemiparetic stroke. Stroke. 1997;28(12):2518-2527.

45. Pineiro R, Pendlebury S, Joharsen-Berg H, Matthews PM. Functional MRI detects posterior shifts in primary sensorimstor certex activation after stroke: evidence of local adaptive reorganization? Stroke. 2001;3/2:5):1134-1139. http://www.ncbi.nlm nih.goy/pubmed/11340222.

46. Ward NS Erown MM, Thompson AJ, Frackowiak RS. Neural correlates of motor recovery after stroke: a longitudinal fMRI study. Brain. 2003;126(Pt 11):2476-2496. doi:awg245 [pii] 10.1e93 brain/awg245.

47 Caryalho SMF, Pontes-Neto OM, Fabio SRC, Leite JP, Santos AC, Araujo DB de. Rapid BOLD fMRI signal loss in the primary motor cortex of a stroke patient. Arq Neuropsiquiatr.

2008;66(4):885-887. doi:10.1590/S0004-282X2008000600022.

48. Veldsman M, Cumming T, Brodtmann A. Beyond BOLD: Optimizing functional imaging in stroke populations. Hum Brain Mapp. 2015;36(4):1620-1636. doi:10.1002/hbm.22711. 


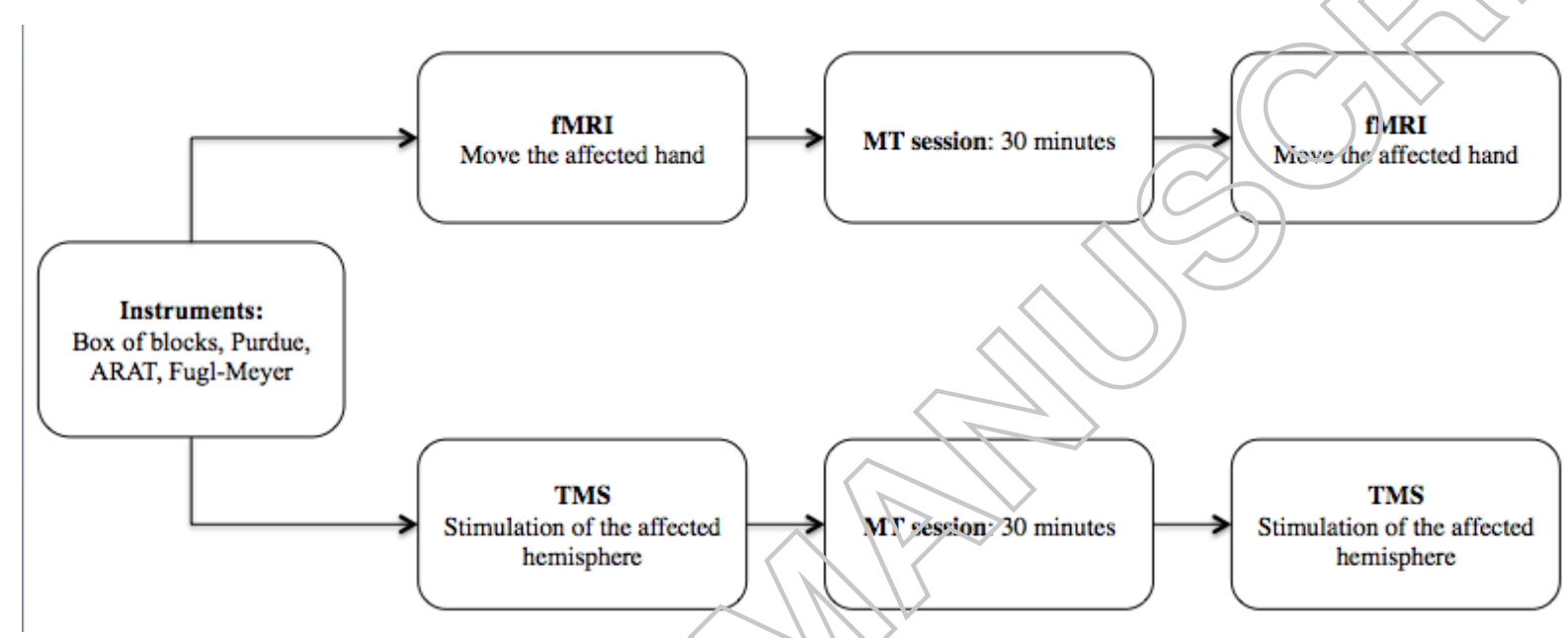

Fig. 1. Experimental design. Clinical, fMRI and TMS evaluations were performed on three different days. At day one, patierts were submitted to a clinical evaluation (Box of blocks, Purdue, ARAT and Fugl-Meyer tests) and were randomly assigned to the order of the assessments (fMRI or TMS). Each assessment (fMRI and TMS) was composed of: i) the (TMS or fMRI) evaluation immediateiy before the MT session, ii) the MT session (30 minutes), and iii) the (TMS or fMKJ eveluatien immediately after the MT session. 

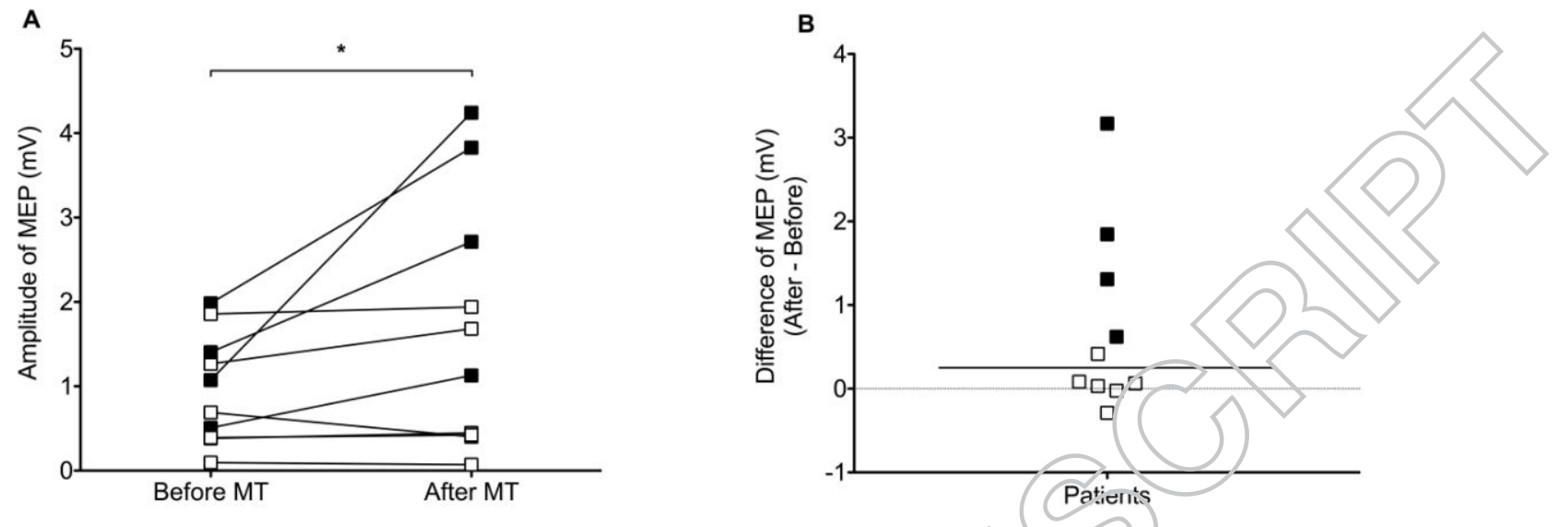

Fig. 2. MT-induced MEP changes. Motors Evoked Potentia!s (MEP) amplitude of the affected hand on the group of patients before and after therapy. (a) MEP median values of all participants before and after MT $\left({ }^{*} \mathrm{p}<0.05\right.$; paired two tailed Wilcoxon test; $\left.N=10\right)$. (b) Individual subject MEP changes (MEP after - MEP before) of all individuals. The solic horizontal line represents the mean. MEP amplitude in the affected hand of four participants (marked in black) increased significantly after therapy ( $\mathrm{p}<0.05$, Mann Whitney test) 
before MT
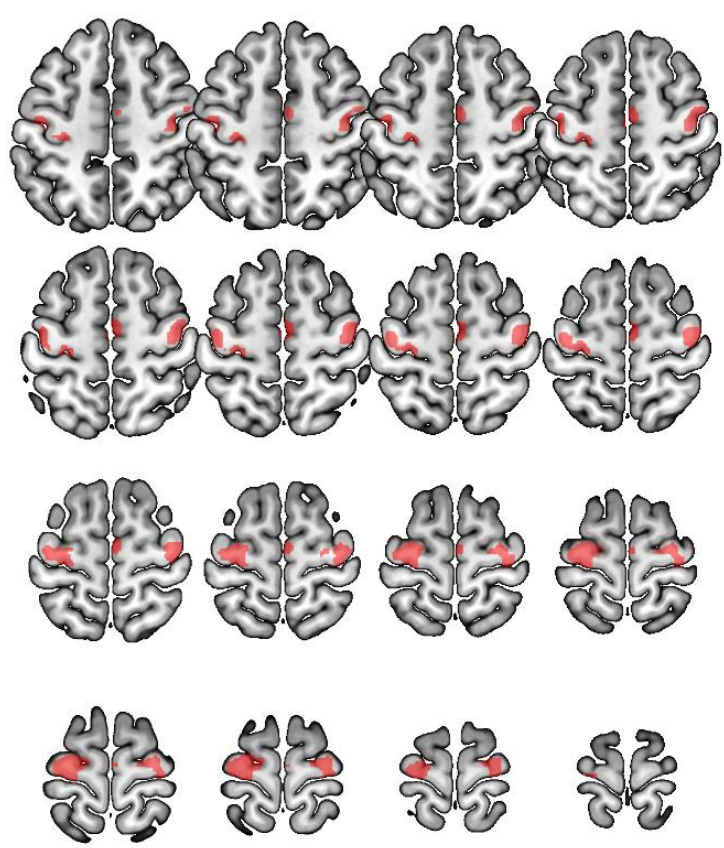

after MT
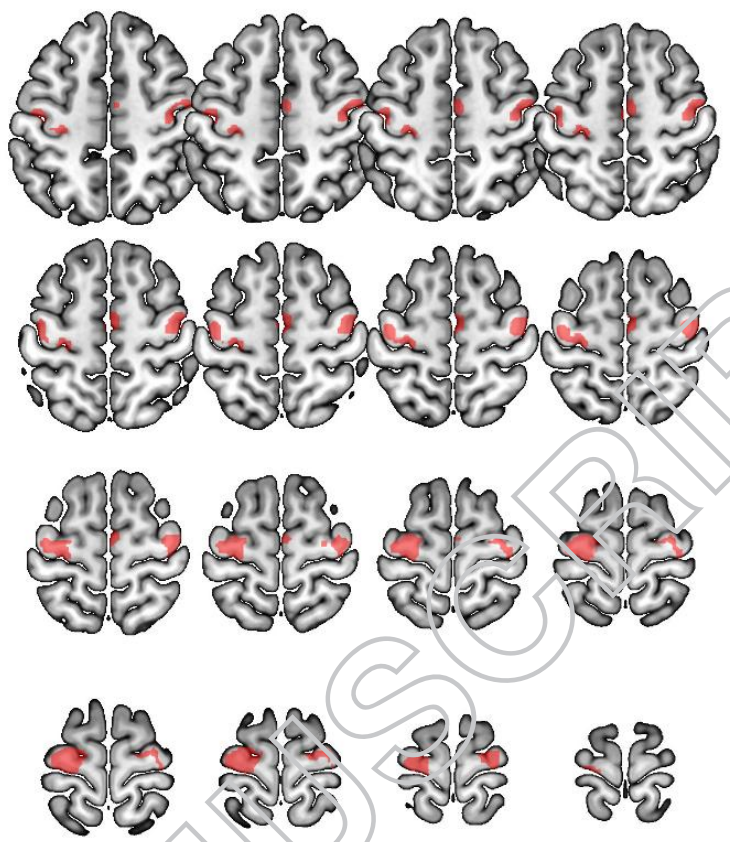

Fig. 3. Group response to the fMRI motor task. The proposed metor task elicited significant activity in bilateral M1 and SMA with asymmetrical (pvoxel $<0.001$, FWE eorrected). 

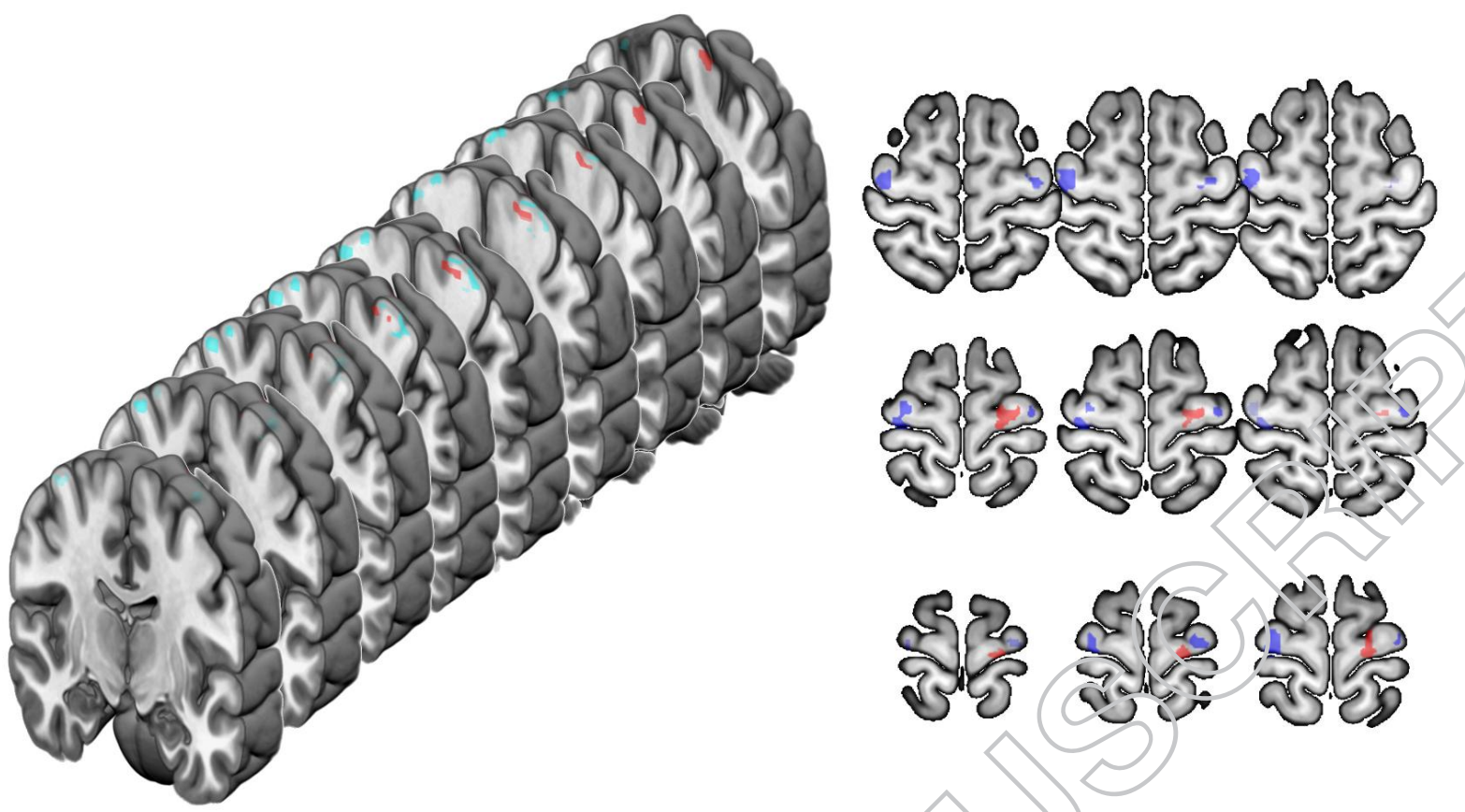

Fig. 4. Between session comparison (Post-MT vs Pre-MT). Cluster within affected M1 showed increased activity (in red), and cluster within bila era! $M$ l showed decreased activity (in blue). pvoxel<0.05, uncorrected, and clusters with p<0.01 at peak level. $\mathrm{N}=13$. 
TMS $x$ fMRI

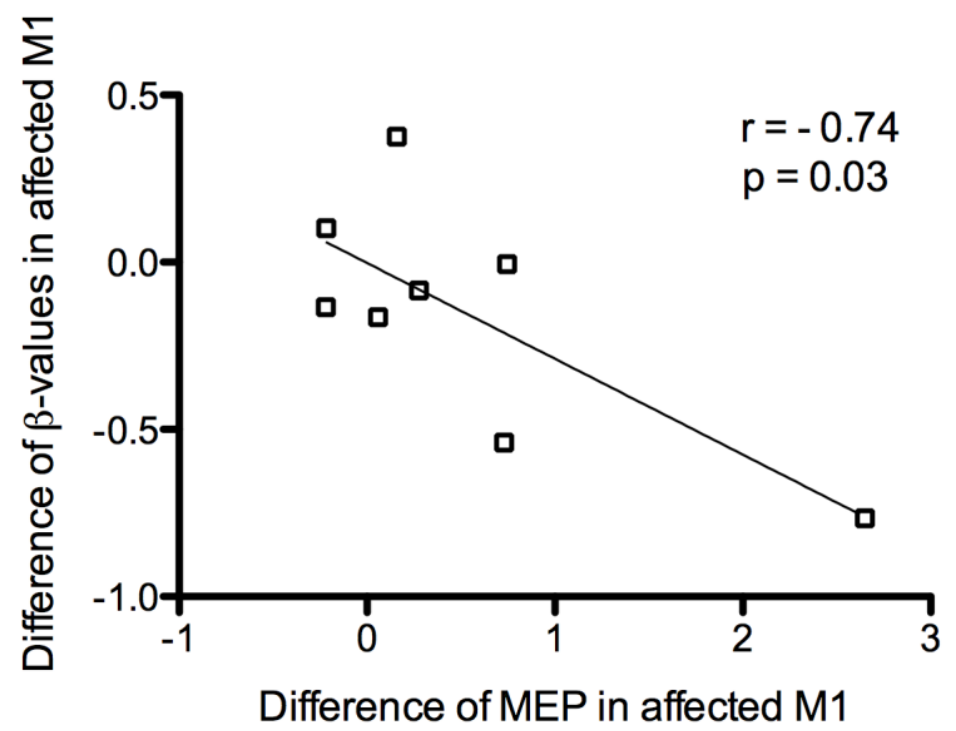

Fig. 5. Pearson's correlation between changes of ?-values and MEP mean amplitude. Values are represented as individual ?-values changes ((after-before)/berore) and the mean amplitude MEP change ((after - before)/before) of the affected M1 Signiticant inverse correlation was found $(\mathrm{r}=$ $0.74, \mathrm{p}=0.03, \mathrm{~N}=8)$. 
Table 1. Demographic data and clinical characteristics (box and block test, ARAT, Fugl-Meyer and Purdue) of all participants.

\begin{tabular}{|c|c|}
\hline NOMINAL VARIABLE & NUMBER OF PARTICIPANTS (\%) \\
\hline \multicolumn{2}{|l|}{ Sex } \\
\hline Male & $10(67 \%)$ \\
\hline Female & $5(33 \%)$ \\
\hline \multicolumn{2}{|l|}{ Affected hemisphere } \\
\hline Left & $8(53 \%)$ \\
\hline Right & \\
\hline \multicolumn{2}{|l|}{ Affected cerebral artery } \\
\hline Middle cerebral artery & \\
\hline Internal carotid artery & \\
\hline Vertebral artery & $1(7 \%)$ \\
\hline
\end{tabular}

Manual dominance

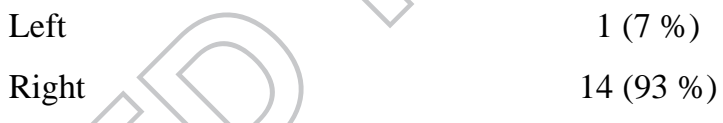

Cognition

Deficit 2(13\%)

No deficit $13(87 \%)$

CONFINUOUS VARIABLE $\quad$ MEAN $\pm \mathrm{SD}$

Age (year) $58 \pm 10$

Time after stroke (months) $25 \pm 22$

$\begin{array}{lccc}\text { MOTOR IMPAIRMENT } & \text { Dif. median } & \mathrm{Z} & \mathrm{P} \\ \text { Box and block test } & 10 & -1.79 & 0.07 \\ \text { ARAT } & 0 & -1.83 & 0.07 \\ \text { Fugl-Meyer } & 2 & -3.06 & 0.002^{* *} \\ \text { Purdue } & 3 & -2.29 & 0.02^{*}\end{array}$

Statistical significance were found in the Fugl-Meyer $(* * \mathrm{p}<0.005)$ and Purdue $(* \mathrm{p}<0.05)$. Dif. Median $=$ difference in median. Wilcoxon two-tailed, $\mathrm{N}=15$. 
Table 2. Personal and clinical characteristics of all participants.

\begin{tabular}{|c|c|c|c|c|c|c|c|c|c|c|c|c|c|c|}
\hline $\begin{array}{l}\text { Sub } \\
\text { j. }\end{array}$ & $\begin{array}{l}\text { Gend } \\
\text { er }\end{array}$ & $\begin{array}{l}\mathrm{Ag} \\
\mathrm{e}\end{array}$ & $\begin{array}{l}\text { Time } \\
\text { since } \\
\text { stroke } \\
\text { (mont } \\
\text { hs) }\end{array}$ & $\begin{array}{l}\text { Affect } \\
\text { ed } \\
\text { Hem. }\end{array}$ & $\begin{array}{l}\text { Manual } \\
\text { Domina } \\
\text { nce }\end{array}$ & $\begin{array}{l}\text { CT \& } \\
\text { MRI } \\
\text { Affect } \\
\text { ed } \\
\text { artery }\end{array}$ & $\begin{array}{l}\text { AR } \\
\text { AT / } \\
\text { A }\end{array}$ & $\begin{array}{l}\mathrm{F} \\
\mathrm{M} \\
/ \\
\mathrm{A}\end{array}$ & $\begin{array}{l}\mathrm{BB} \\
\mathrm{T} / \\
\mathrm{U}\end{array}$ & $\begin{array}{l}\mathrm{BB} \\
\mathrm{T} / \\
\mathrm{A}\end{array}$ & $\begin{array}{l}\text { Purd } \\
\text { ue / } \\
\text { U }\end{array}$ & $\begin{array}{l}\text { Purd } \\
\text { ue/ A }\end{array}$ & $\begin{array}{l}\text { First } \\
\text { assessm } \\
\text { ent }\end{array}$ & $\begin{array}{l}\text { Second } \\
\text { assessin } \\
\text { ent }\end{array}$ \\
\hline 1 & $\mathrm{M}$ & 60 & 24 & $\mathrm{R}$ & $\mathrm{R}$ & $\mathrm{MCA}$ & 57 & 76 & 49 & 38 & 9.33 & 7.67 & TMS & $\mathrm{fNiRI}$ \\
\hline 2 & $\mathbf{M}$ & 59 & 7 & $\mathbf{R}$ & $\mathbf{R}$ & $\begin{array}{l}\text { MCA } \\
\text { and } \\
\text { ACA }\end{array}$ & 57 & 76 & 32 & 36 & $\begin{array}{l}11.3 \\
3\end{array}$ & 11.33 & & fMIR \\
\hline 3 & $\mathrm{M}$ & 47 & 41 & $\mathrm{~L}$ & $\mathrm{R}$ & MCA & 57 & 70 & 43 & 41 & $\begin{array}{l}10.6 \\
7\end{array}$ & 5.67 & $\mathrm{fN}: \mathrm{RI}$ & TMS \\
\hline 4 & $\mathbf{M}$ & 54 & 19 & $\mathbf{R}$ & $\mathbf{R}$ & ICA & 57 & 72 & 41 & 40 & 7.67 & 8.23 & TMS & fMRI \\
\hline 5 & $\mathrm{M}$ & 65 & 24 & $\mathrm{~L}$ & $\mathrm{R}$ & MCA & 57 & 69 & 54 & 38 & 10.6 & 7.01 & fMRI & TMS \\
\hline 6 & $\mathbf{M}$ & 60 & 36 & $\mathbf{R}$ & $\mathbf{R}$ & MCA & 57 & 76 & 60 & $5 \varepsilon$ & 13 & 10.67 & TMS & fMRI \\
\hline 7 & $\mathrm{~F}$ & 76 & 11 & $\mathrm{~L}$ & $\mathrm{R}$ & MCA & 57 & 74 & 27 & 31 & 5.67 & 8.67 & fMRI & TMS \\
\hline 8 & $\mathrm{~F}$ & 59 & 22 & $\mathrm{~L}$ & $\mathrm{R}$ & MCA & 57 & 75 & 68 & 15. & 13 & 13,67 & fMRI & TMS \\
\hline 9 & $\mathbf{M}$ & 68 & 32 & $\mathbf{L}$ & $\mathbf{R}$ & MCA & 57 & 74 & 47 & 53 & 3.67 & 10.67 & TMS & fMRI* \\
\hline 10 & $\mathrm{~F}$ & 34 & 96 & $\mathrm{~L}$ & $\mathrm{R}$ & MCA & 57 & $7+$ & 65 & $6 ?$ & $\begin{array}{l}11.6 \\
7\end{array}$ & 15.33 & TMS* & fMRI \\
\hline 11 & $\mathrm{M}$ & 58 & 13 & $\mathrm{~L}$ & $\mathrm{R}$ & $\begin{array}{l}\text { Verteb } \\
\text { ral } \\
\text { artery }\end{array}$ & 3 & & 65 & 0 & 12 & 0 & fMRI & TMS* \\
\hline 12 & $\mathrm{~F}$ & 56 & 12 & $\mathrm{~L}$ & $\mathrm{R}$ & MCA & $5 \%$ & 5 & 48 & 43 & $\begin{array}{l}12.6 \\
7 \\
\end{array}$ & 8.33 & TMS* & fMRI \\
\hline 13 & $\mathrm{M}$ & 64 & 6 & $\mathrm{R}$ & $\mathrm{R}$ & ICA & 56 & 72 & 47 & 39 & 10 & 6.33 & fMRI & TMS* \\
\hline 14 & $\mathrm{M}$ & 62 & 14 & $\mathrm{R}$ & $\mathrm{L}$ & $\overline{\mathrm{MCA}}$ & 45 & 60 & 57 & 16 & 12 & 1 & TMS* & fMRI \\
\hline 15 & $\mathrm{~F}$ & 54 & 14 & $\mathrm{R}$ & $\mathrm{R}$ & & 48 & 64 & 62 & 27 & $\begin{array}{l}11.3 \\
3\end{array}$ & 5.33 & TMS & fMRI* \\
\hline
\end{tabular}

$\mathrm{M}=$ male, $\mathrm{F}=$ female, $\mathrm{P}=$ righ, $\mathrm{V}=\mathrm{left}, \mathrm{MCA}=$ Middle Cerebral Artery, ICA = Internal Carotid Artery, ACA = Anterior Cerebral Artery, FM=Fugl-Meyer, BBT=Box and block test. Participants with significant TMIS changes are represented in bold. *data was not considered for statistical analysis. 
Table 3. Individual between-session (post-MT - pre-MT) Motor Evoked Potentials (MEP).

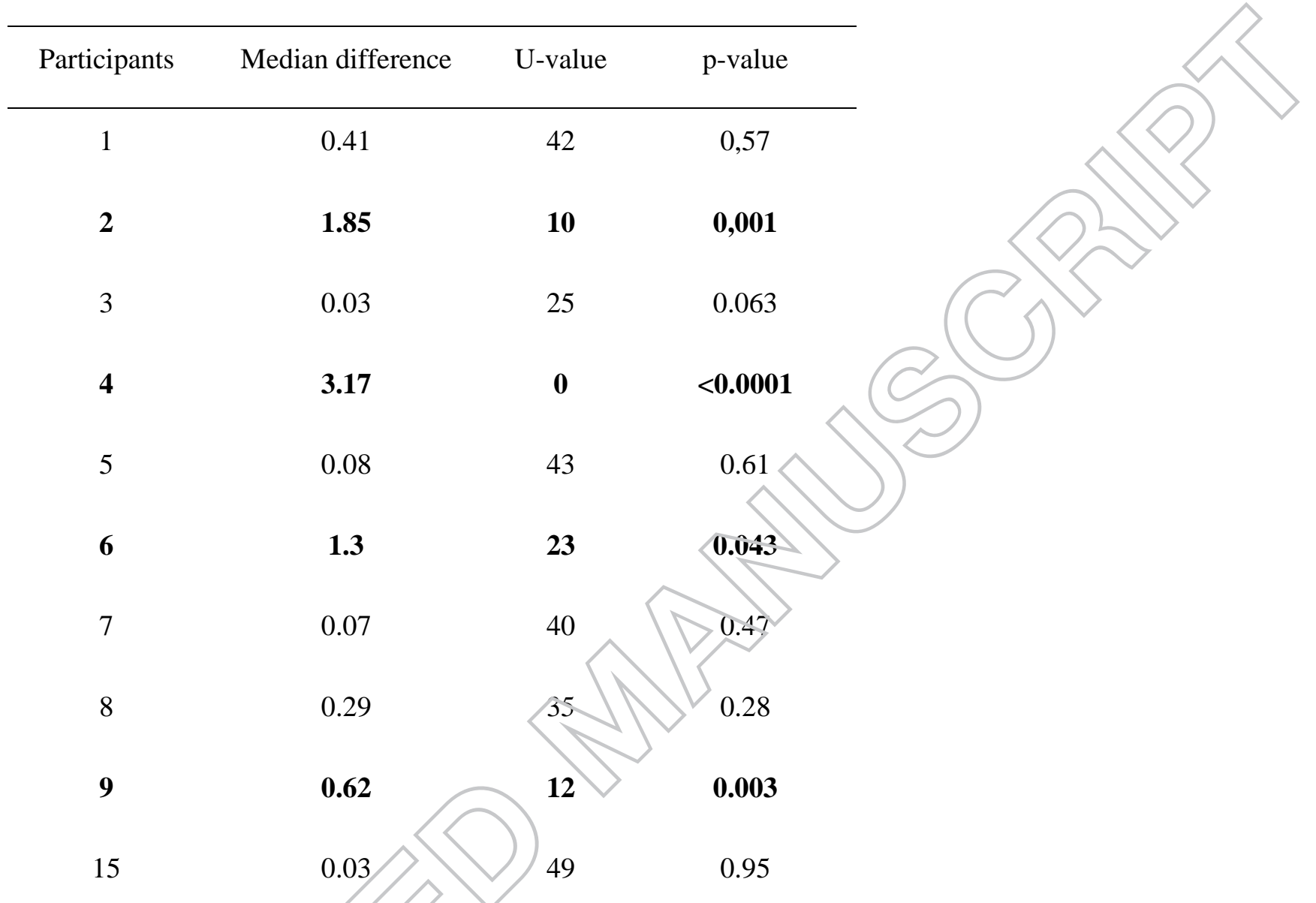

Participants with significant TMS changes are represented in bold. The 10 detected MEPs for each subject were compared (pre-MT vs post-MT) using the Mann-Whitney test. We expressed values as median, U-value and statisticai significance was set at $\mathrm{p}<0.05$ 